\title{
Quantum treatment of Verlinde's entropic force conjecture
}

\author{
A. Plastino ${ }^{1,4,5}$, M. C. Rocca ${ }^{1,2,4}$, G. L. Ferri ${ }^{3}$, \\ ${ }^{1}$ Departamento de Física, Universidad Nacional de La Plata, \\ 2 Departamento de Matemática, Universidad Nacional de La Plata, \\ ${ }^{3}$ Fac. de C. Exactas-National University La Pampa, \\ Peru y Uruguay, Santa Rosa, La Pampa, Argentina \\ ${ }^{4}$ Consejo Nacional de Investigaciones Científicas y Tecnológicas \\ (IFLP-CCT-CONICET)-C. C. 727, 1900 La Plata - Argentina \\ ${ }^{5}$ SThAR - EPFL, Lausanne, Switzerland
}

August 7, 2018

\begin{abstract}
Verlinde conjectured that gravitation is an emergent entropic force. This surprising conjecture was proved in [Physica A 505 (2018) 190] within a purely classical context. Here, we appeal to a quantum environment to deal with the conjecture in the case of bosons and consider also the classical limit of quantum mechanics (QM).
\end{abstract}

Keywords: Gravitation, bosons, entropic force, emergent force, Verlide's conjecture.. 


\section{Contents}

1 Introduction

2 Entropic force for bosons 3

2.1 Quantum entropic force ..................... 3

2.2 Bosonic entropic force in the classical limit (CL) . . . . . . 5

2.3 Entropic Potential Energy . . . . . . . . . . . 6

3 Conclusions 7 


\section{Introduction}

Eight years ago, Verlinde [1] proposed to link gravity with an entropic force. The ensuing conjecture was proved recently [2], in a purely classical environment.

According to Verlinde, gravity would emerge as a result of information about the positions of material particles, connecting a thermal treatment of gravity to 't Hooft's holographic principle. In this perspective, gravitation should be regarded as an emergent phenomenon. This Verlinde's idea was the focus of much attention. For an example, see [3, 4]. An excellent overview on the statistical mechanics of gravitation can be found in Padmanabhan's article [5], and references therein.

Verlinde's work originated endeavors in cosmology, the dark energy hypothesis, cosmological acceleration, cosmological inflation, and loop quantum gravity. The associated literature is extensive [4]. An important contribution is that of Guseo [6]. He showed that the local entropy function, related to a logistic distribution, is a catenary and vice versa, an invariance that may be interpreted through Verlinde's conjecture regarding gravity's origin as an entropic force. Guseo advances a novel interpretation of the local entropy in a system [6].

This paper does not treat any of these issues, though. Based on the fact that we proved Verlinde's hypothesis in a classical environment [2], we wish to deal here with the quantal bosonic scenario.

\section{Entropic force for bosons}

\subsection{Quantum entropic force}

The Bose gas' entropy is (see, for instance, [7])

$$
\mathcal{S}=N k_{B}\left[\left(\frac{n}{N}\right) \ln \left(1+\frac{N}{n}\right)+\ln \left(1+\frac{n}{N}\right)\right],
$$

where

$$
n=V\left(\frac{E}{N}\right)^{\frac{3}{2}}\left(\frac{4 \pi e m}{2 h^{2}}\right)^{\frac{3}{2}}
$$


Since we can cast the volume as

$$
V=\frac{4}{3} \pi r^{3},
$$

we recast (2.1) as

$$
\begin{gathered}
\mathcal{S}=k_{B} V\left(\frac{E}{N}\right)^{\frac{3}{2}}\left(\frac{4 \pi e m}{2 h^{2}}\right)^{\frac{3}{2}} \ln \left[1+\frac{N}{V}\left(\frac{N}{E}\right)^{\frac{3}{2}}\left(\frac{2 h^{2}}{4 \pi e m}\right)^{\frac{3}{2}}\right]+ \\
N k_{B} \ln \left[1+\frac{V}{N}\left(\frac{E}{N}\right)^{\frac{3}{2}}\left(\frac{4 \pi e m}{2 h^{2}}\right)^{\frac{3}{2}}\right] .
\end{gathered}
$$

Now, according to [2] the entropic force is

$$
\begin{gathered}
F_{e}=-\lambda \frac{\partial S}{\partial A}= \\
\frac{\lambda 3 k_{B} N}{8 \pi r^{2}} \frac{1}{1+\frac{3 N}{4 \pi r^{3}}\left(\frac{N}{E}\right)^{\frac{3}{2}}\left(\frac{3 h^{2}}{4 \pi e m}\right)^{\frac{3}{2}}}- \\
\frac{\lambda k_{B}}{2}\left(\frac{E}{N}\right)^{\frac{3}{2}}\left(\frac{4 \pi e m}{2 h^{2}}\right)^{\frac{3}{2}} r \ln \left[1+\frac{3 n}{4 \pi r^{3}}\left(\frac{N}{E}\right)^{\frac{3}{2}}\left(\frac{3 h^{2}}{4 \pi e m}\right)^{\frac{3}{2}}\right]- \\
\frac{\lambda k_{B}\left(\frac{E}{N}\right)^{\frac{3}{2}}\left(\frac{4 \pi e m}{2 h^{2}}\right)^{\frac{3}{2}} r}{1+\frac{4 \pi r^{3}}{3 N}\left(\frac{E}{N}\right)^{\frac{3}{2}}\left(\frac{4 \pi e m}{2 h^{2}}\right)^{\frac{3}{2}}},
\end{gathered}
$$

where $\lambda$ is an arbitrary constant. We can recast the above expression as

$$
\begin{gathered}
F_{e}=\frac{12 \lambda k_{B} N(\pi e m E)^{\frac{3}{2}} r}{32 \pi r^{3}(\pi e m E)^{\frac{3}{2}}+3^{\frac{5}{2}} N^{\frac{5}{2}} h^{3}}- \\
\frac{4 \pi \lambda k_{B}(\pi e m E)^{\frac{3}{2}}}{(3 N)^{\frac{3}{2}} h^{3}} r\left\{\ln \left[32 \pi r^{3}(\pi e m E)^{\frac{3}{2}}+(3 N)^{\frac{5}{2}} h^{3}\right]-\ln \left[32 \pi r^{3}(\pi e m E)^{\frac{3}{2}}\right]\right\}- \\
\frac{12 \lambda k_{B} N(\pi e m E)^{\frac{3}{2}} r}{32 \pi r^{3}(\pi e m E)^{\frac{3}{2}}+(3 N)^{\frac{5}{2}} h^{3}},
\end{gathered}
$$

and then

$$
F_{e}=\frac{4 \pi \lambda k_{B}(\pi e m E)^{\frac{3}{2}}}{(3 N)^{\frac{3}{2}} h^{3}} r\left\{\ln \left[32 \pi r^{3}(\pi e m E)^{\frac{3}{2}}+(3 N)^{\frac{5}{2}} h^{3}\right]-\ln \left[32 \pi r^{3}(\pi e m E)^{\frac{3}{2}}\right]\right\} .
$$




\subsection{Bosonic entropic force in the classical limit (CL)}

The CL is attained for [7]

$$
\frac{N}{n}<<1
$$

and in this limit the entropy becomes [7]

$$
\mathcal{S}=N k_{B}\left[1+\ln \left(\frac{n}{N}\right)\right]
$$

or

$$
\mathcal{S}=\frac{5 N k_{B}}{2}+N k_{B} \ln \left[\frac{V}{N}\left(\frac{E}{N}\right)^{\frac{3}{2}}\left(\frac{4 \pi m}{2 h^{2}}\right)^{\frac{3}{2}}\right] .
$$

Now, we have an entropic force of the form

$$
F_{e}=-\lambda \frac{\partial S}{\partial A}=-\frac{\lambda 3 N k_{B}}{8 \pi r^{2}}
$$

which is indeed of the Newton appearance, so that Verlinde's conjecture gets proved in the classical limit. Note also that the entropic force (2.11) can be derived as well from (2.7) by taking $r$ large enough.

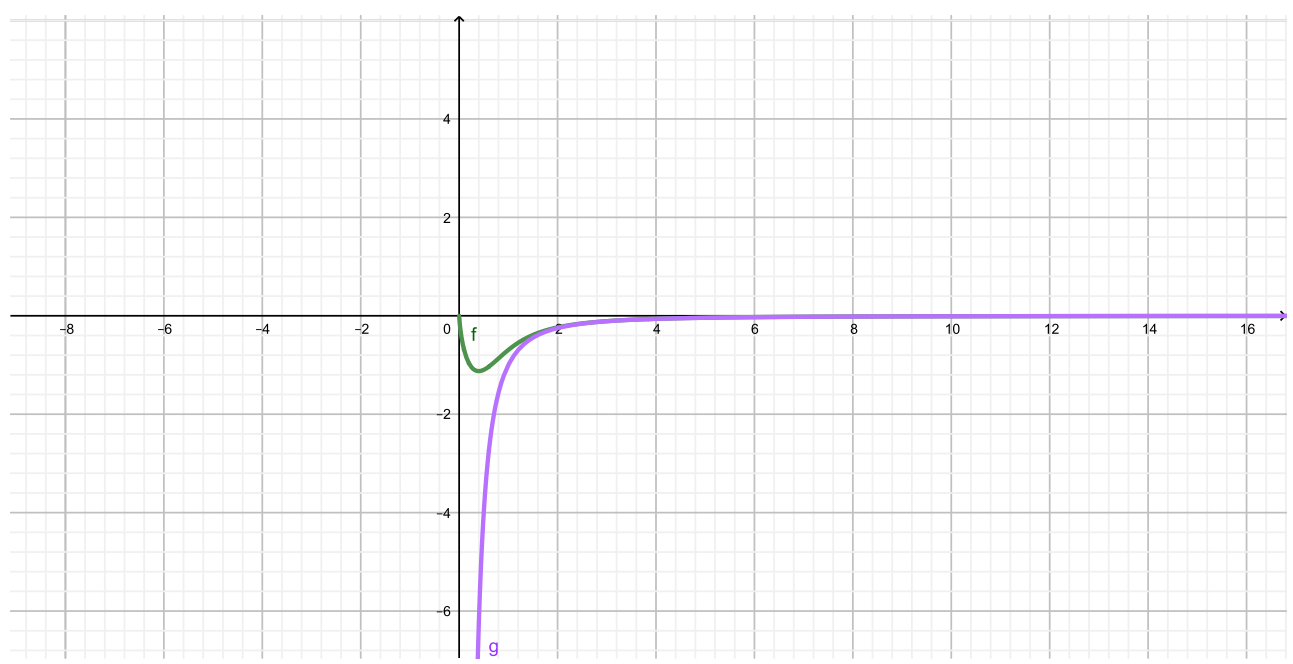

Figure 1: Here we plot $F_{e} / C, C=\frac{3 N k_{B} \lambda}{8 \pi}$. Green line: Boson entropic force. Violet line: approximate semi-classic Bose-one. Here, on the $\mathrm{x}$ axis, 1 unit $=10^{-37}$ meter and, on the y axis, 1 unit $=10^{-4}$ Newton 
In Fig. 1 we set: $m=$ the oxygen-molecule's mass, $E=\frac{N m v^{2}}{2}, v=1$ meter $/$ second, and $N$ is extracted from

$$
32 \pi(\pi e m E)^{\frac{3}{2}}=(3 N)^{\frac{5}{2}} h^{3}
$$

, so that $N=2.358458 \times 10^{26}$. The classic entropic force (2.11) can also be encountered starting from (2.7) and taking $r$ large enough. Appealing such $r$ values and using the equality

$$
-\frac{\lambda 3 N k_{B}}{8 \pi r^{2}}=-\frac{G m M}{r^{2}}
$$

where $G$ is the gravitational constant, we detect the fact that $\lambda=\lambda(m, M, N)$.

\subsection{Entropic Potential Energy}

The entropic force is proportional to the derivative of the entropy with respect to the spherical area $A$. It is interesting to calculate the corresponding potential energy $E_{P}$. To such an end we define the new constants $a$ and $b$ in the fashion $a=(3 N)^{\frac{5}{2}} h^{3}$ and $b=32 \pi(\pi e m E)^{\frac{3}{2}}$. Using reference [8] we can compute the potential energy we are looking for from the entropic force's expression. The ensuing calculation is simple but lengthy. One arrives at the result

$$
\begin{gathered}
E_{P}(r)=\frac{3 N k_{B} \lambda}{8 \pi} \frac{b}{a}\left\{\frac{r^{2}}{2} \ln \left(1+\frac{a}{b r^{3}}\right)-\frac{a^{\frac{2}{3}}}{2 b^{\frac{2}{3}}}\left\{\frac{1}{2} \ln \left[\frac{\left[r+\left(\frac{a}{b}\right)^{\frac{1}{3}}\right]^{2}}{r^{2}-\left(\frac{a}{b}\right)^{\frac{1}{3}} r+\left(\frac{a}{b}\right)^{\frac{2}{3}}}\right]+\right.\right. \\
\left.\left.\sqrt{3}\left[\frac{\pi}{2}-\arctan \left[\frac{2 r-\left(\frac{a}{b}\right)^{\frac{1}{3}}}{\sqrt{3}\left(\frac{a}{b}\right)^{\frac{1}{3}}}\right]\right]\right\}\right\}
\end{gathered}
$$

where we have set $E_{P}(r)=0$ for $r \rightarrow \infty$. For $r$ large the potential energy adopts the appearance

$$
E_{P}(r)=-\frac{\lambda 3 N k_{B}}{8 \pi r}
$$

which is consistent with the result (2.11). 


\section{Conclusions}

We have here considered Verlinde's [entropic force - Gravitation] link, proved recently in a classical context [2], in a quantum bosonic scenario. We have seen that Verlinde's conjecture holds in this scenario as well. Further, the quantum emergent gravitation à la Verlinde does not diverge at the origin. Such an asymptotic behaviour is rather surprising. One wonders whether this emergent gravitation-behaviour might perhaps be an artifact of not being able to include general relativity effects. Moreover, in two limits

- the classical limit of QM

- $r \rightarrow \infty$,

the Newton $r$-dependence of the gravitation force is recovered. A natural challenge is to attack the fermionic case. This we did in reference [9]. For fermions, the vanishing of the entropic force at the origin is also observed. 


\section{References}

[1] E. Verlinde, arXiv:1001.0785 [hep-th]; JHEP 04 (2011) 29.

[2] A. Plastino, M. C. Rocca: Physica A 505 (2018) 190.

[3] D. Overbye, A Scientist Takes On Gravity, The New York Times, 12 July 2010; M. Calmthout, New Scientist 205 (2010) 6.

[4] J. Makela, arXiv:1001.3808v3; J. Lee, arXiv:1005.1347; V. V. Kiselev, S. A. Timofeev , Mod. Phys. Lett. A 25 (2010) 2223; T. Aaltonen et al; Mod. Phys. Lett. A 25 (2010) 2825.

[5] T. Padmanabhan, arXiv 0812.2610v2.

[6] R. Guseo, Physica A 464 (2016) 1.

[7] D. S. Lemons:"A Student's Guide to Entropy". Cambridge University Press (2014).

[8] I. S. Gradshteyn and I. M. Ryzhik : "Table of Integrals, Series and Products". Academic Press, Inc (1980).

[9] https://www.researchgate.net/publication/324831972_Spatial_cut-offs_Fermion_St 